\title{
Soluble Tumor Necrosis Factor Receptor Superfamily Member 8
}

National Cancer Institute

\section{Source}

National Cancer Institute. Soluble Tumor Necrosis Factor Receptor Superfamily Member

8. NCI Thesaurus. Code C103890.

Soluble tumor necrosis factor receptor superfamily member $8(\sim 85 \mathrm{kDa})$ is encoded by the human TNFRSF8 gene. This enzymatically processed protein may be involved in the inhibition of tumor necrosis factor lig and superfamily member 8 (CD30 lig and) binding to its cell surface-localized receptor. 